\title{
Construcción de un banco para prácticas electroneumáticas
}

\author{
Construction of a bench for electro-pneumatic practices
}

\author{
Paolo Amato Hernández ${ }^{1}$, Mario Camargo Ardila ${ }^{1}$, Jhonatan Durán Bastidas ${ }^{1}$, Carlos Pacheco \\ Amell $^{1}$, Javier Roldán Mckinley ${ }^{1}$, Jovanny Duque ${ }^{2}$ \\ ${ }^{1}$ Ingeniería Mecánica, Universidad de Atlántico, Barranquilla Colombia. \\ javierroldan@mail.uniatlantico.edu.co \\ ${ }^{1}$ Ingeniería Mecánica, Instituto Tecnológico de Soledad, Barranquilla; Costa Atlántica; Colombia \\ jduquelitsa.edu.co
}

\begin{abstract}
Resumen - Se presentan los fundamentos para la construcción de un banco de pruebas de entrenamiento para prácticas electroneumáticas. Se detallan los aspectos indispensables para su diseño, tales como la ergonomía, el análisis de cargas, la selección de elementos estructurales y adaptación de elementos neumáticos, para obtener una adecuada estructura de trabajo. La utilidad $y$ practicidad del banco en su ensamble $y$ desensamble fue mostrada en la ejecución de las prácticas de laboratorio diseñadas, $y$ complementadas con material audiovisual para instructores y estudiantes.
\end{abstract}

Palabras clave - electroneumática; banco de pruebas; diseño; práctica de laboratorio.

Abstract-Construction criteria for an electro-pneumatic teaching bench are presented. A suitable work bench was obtained based on Ergonomics, load analysis and structural elements selection. Pneumatic circuit elements were modified to fit into the bench work area. The usefulness in the assembly for the created lab practices was highlighted, and complemented with audiovisual aids for both instructors and students.

Key Word - electro-pneumatic; design; test bench; lab practice.

\section{INTRODUCCIÓN}

Dentro de las competencias que corresponden a un ingeniero mecánico está el dominio de la automatización neumática e hidráulica, por lo cual los fundamentos teóricos de los sistemas neumáticos y su combinación con un sistema electrónico son esenciales para el desarrollo de una buena praxis [1].

Actualmente, el tiempo de producción y la calidad de los productos es un aspecto muy importante para el campo industrial, por este motivo cada día se incrementa la aceleración del avance de las ciencias y el desarrollo tecnológico. Debido a este avance se ha recurrido a reemplazar la lógica cableada en los sistemas de automatización de contactos por la lógica programada, ya que un proceso automatizado brinda mejores garantías de calidad y competitividad. Se requiere entonces que el ingeniero mecánico egrese con conocimientos teóricos y prácticos de la simbología de circuitos neumáticos [2, 3] y sus elementos: compresores, redes de aire, tanques de almacenamiento, limpieza y adecuación del aire, válvulas, tipos y características de sensores, circuitos neumáticos básicos, etc..., además de su puesta en marcha y mantenimiento.

En este documento se encuentran los detalles relacionados al diseño y construcción de un banco de pruebas electroneumáticas como recurso para la enseñanza de las asignaturas relacionadas con Instrumentación Industrial, Automatización, Controladores Lógicos Programables, Hidráulica y Neumática, del Programa de Ingeniería Mecánica de la Universidad _de Atlántico. En el aspecto funcional, el equipo de pruebas permitirá fácil ensamble y desensamble de los circuitos neumáticos de las experiencias para permitir a los estudiantes diseñar rápidamente el circuito de prueba, pudiendo así diseñar más de un experimento por sesión de laboratorio. Se tendrán en cuenta aspectos de comodidad y ergonomía al fundamentar el diseño del equipo en las indicaciones pertinentes de diseño de producto. El principal objetivo es brindar herramientas educativas para que el estudiante, por medio de la práctica, enfrente los problemas y situaciones más comunes que se deberán solucionar una vez ingrese a la práctica real.

\section{ASPECTOS DE DISEÑO}

\section{A. Ergonomía del banco.}

Uno de los principales criterios que se tienen en cuenta en el desarrollo del proyecto es la ergonomía. Ésta se define como la disciplina científica que se relaciona con la comprensión de las interacciones entre humanos y otros elementos de un sistema, igualmente, como la profesión que busca la optimización del bienestar de las personas y el rendimiento 
global del sistema aplicando teoría, principios, datos y métodos para el diseño [4]. Se utilizará la ergonomía para adaptar el producto y el entorno a las dimensiones, capacidades y necesidades de los estudiantes e instructores, para así aumentar factores como eficiencia, seguridad y bienestar en los usuarios finales del producto [5]. Este aspecto es de vital importancia en el diseño del banco de trabajo, pues así se asegura que el personal involucrado en el uso del componente no sufra fatigas y/o lesiones musculares producto de esfuerzos innecesarios.

\section{B. Diseño básico.}

El correcto diseño del banco de trabajo es importante por seguridad y eficiencia al momento del desarrollo de la actividad a realizar. Un diseño adecuado del banco de trabajo conlleva a que el usuario pueda conservar una postura corporal correcta y agradable, por el contrario una postura laboral incómoda puede ocasionar múltiples problemas físicos. Entre los puntos críticos del banco, en cuanto a ergonomía se refiere, se encuentran: la altura de la superficie de trabajo, la dimensión del área de trabajo y la altura máxima del banco.

La antropometría se define como la disciplina que describe las diferencias cuantitativas de las medidas del cuerpo humano, estudia las dimensiones tomando como referencia distintas estructuras anatómicas, y sirve de herramienta a la ergonomía con objeto de adaptar el entorno a las personas [6]. Diseñar el puesto de trabajo para que se ajuste a los individuos en general es la guía primordial, pero en el sentido práctico pocos profesionales de la ergonomía recolectan sus propios datos, pues mucha de esta información ya se encuentra tabulada.

El diseño depende de la población objetivo del mismo, utilizando en este caso el criterio de diseño para el promedio, el cual solamente se utiliza en contadas situaciones, cuando: la precisión de la dimensión tiene poca importancia, no provoca dificultades o su frecuencia de uso es muy baja, cualquier otra solución es o muy costosa, o técnicamente muy compleja [6].

\section{Diseño detallado.}

Aunque no existe un individuo que posea las dimensiones promedio en su totalidad, existen escenarios en los que resulta impráctico o de gran costo incluir posibilidades de ajuste para todas las características. Para empezar el diseño se tienen en cuenta las medidas de la población en Colombia, ya que por lo general al adquirir una máquina, esta es de origen extranjero, lo que se traduce en muchos casos, en dificultades para su manejo, pues los trabajadores deben hacer esfuerzos innecesarios al no estar diseñada para esa población especifica. El mobiliario utilizado en sitios de trabajo es fabricado casi siempre de manera empírica, con dimensiones que no consultan las necesidades del usuario, que en una gran cantidad de ocasiones obligan a posturas incómodas y a esfuerzos indebidos [7].
Colombia no cuenta con una base oficial de datos antropométricos de la población. Las Tablas I y II muestran los parámetros antropométricos de la población laboral colombiana del año 1995, femenina y masculina, respectivamente; de las cuales se toman los datos necesarios para dimensionar el banco.

\begin{tabular}{|l|l|}
\hline Nombre de la variable & $\mathrm{P}_{50}(\mathrm{~cm})^{*}$ \\
\hline Altura radial (parado) & 106,5 \\
\hline Alcance lateral con asimiento & 76,9 \\
\hline Alcance anterior con asimiento & 71,4 \\
\hline Altura acromial (parado) & 137,9 \\
\hline
\end{tabular}

*: Percentil 50

Tabla 1. Medidas antropométricas población laboral colombiana (sexo masculino) [7].

\begin{tabular}{|l|l|}
\hline Nombre de la variable & $\mathrm{P}_{50}(\mathrm{~cm})^{*}$ \\
\hline Altura radial (parado) & 97,8 \\
\hline Alcance lateral con asimiento & 70,1 \\
\hline Alcance anterior con asimiento & 65,6 \\
\hline Altura acromial (parado) & 127,1 \\
\hline
\end{tabular}

*: Percentil 50

Tabla 2. Medidas antropométricas población laboral colombiana (sexo femenino) [7].

\section{Altura de superficie de trabajo.}

Una postura cómoda para el trabajo del operario determina la altura de superficie de trabajo. En general, esto representa una posición natural para los brazos, flexionando los codos a $90^{\circ}$ de manera que los antebrazos estén paralelos al suelo. La dimensión de operación adecuada para la superficie de trabajo se traduce en la altura del codo. Si distancia del piso al codo es muy alta, lo antebrazos tienden a encogerse causando fatiga en los hombros. Si por el contrario es muy baja, el operario se ve obligado a doblar el cuello o la espalda y esto ocasiona fatiga en estos [7].

El tipo de trabajo que se realice determina una altura óptima para la superficie de trabajo. Para trabajo de precisión, la altura de la superficie de trabajo debe ser de 0 a $10 \mathrm{~cm}$ por encima del codo, lo cual sirve de soporte reduciendo las cargas estáticas en los hombros. Para trabajo ligero, la altura de la superficie de trabajo debe de ser de 0 a $10 \mathrm{~cm}$ por debajo del codo para materiales y herramientas pequeñas. Para trabajo pesado, la altura de la superficie de trabajo debe ser de 10 a $20 \mathrm{~cm}$ debajo del codo para permitir un buen trabajo muscular de la extremidad superior [6].

Al determinar la altura adecuada de la superficie de trabajo, es importante tener en cuenta:

- La altura de los codos del trabajador.

- El tipo de trabajo que habrá que desarrollar.

- El tamaño del producto con el que se trabajará.

- Las herramientas y el equipo que se habrán de usar. 
Hay que seguir las siguientes normas para que el cuerpo adopte una buena posición si hay que trabajar de pie:

- Estar frente al producto o la máquina.

- Mantener el cuerpo próximo al producto de la máquina.

- Mover los pies para orientarse en otra dirección en lugar de girar la espalda o los hombros.

Diseñar para la altura de operación del percentil 50 para la altura del codo de las poblaciones de hombres y mujeres combinadas (en términos generales, el promedio de los valores del percentil 50 de hombres y mujeres), significa que la mayoría de los individuos no tendrán inconvenientes serios. Sin embargo, el hombre excepcionalmente alto o la mujer muy baja puede experimentar incomodidad en la postura.

En la Tabla 1 la altura al codo (radial) del percentil 50 para hombres es de $106.5 \mathrm{~cm}$, mientras que para las mujeres (Tabla II) es de $97.8 \mathrm{~cm}$, para obtener $102.15 \mathrm{~cm}$ el promedio de estos dos valores. Siendo trabajo ligero la naturaleza del trabajo a realizar, la superficie de trabajo se determinó a estar $10 \mathrm{~cm}$ por debajo de la altura del codo, teniendo una altura de trabajo de $92 \mathrm{~cm}$ (Fig. 1.a.)

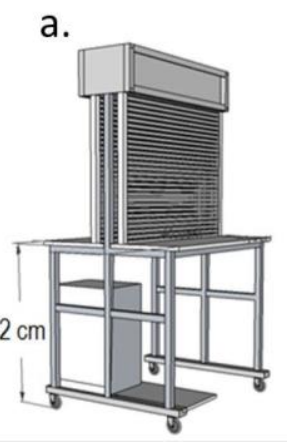

b.

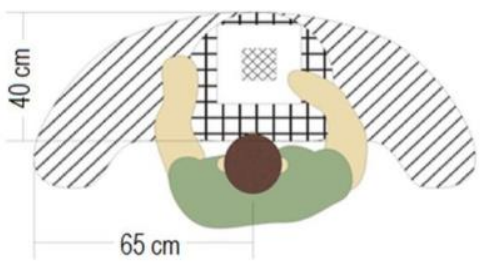

Figura 1. Altura de trabajo (a) y área de trabajo (b).

E. Área de trabajo.

En cada movimiento interviene una distancia y mientras más grande es la distancia, mayores son el esfuerzo muscular, el control y el tiempo requeridos, por lo cual es importante minimizar estas distancias. El área normal de trabajo de la mano derecha en el plano horizontal incluye el área circunscrita por el antebrazo al moverlo en forma de arco con pivote en el codo [4]. Esta área representa la zona más conveniente dentro de la cual la mano realiza movimientos con un gasto normal de energía.

El área normal de la mano izquierda se establece de manera similar. Como los movimientos se hacen en tercera dimensión, al igual que en el plano horizontal, el área normal de trabajo se aplica también al plano vertical. El área normal relativa a la altura para la mano derecha incluye el área circunscrita por el antebrazo en posición hacia arriba con el codo como pivote y moviéndose en un arco. Existe un área normal similar en el plano vertical para el brazo extendido [4].

El espacio de la superficie de la mesa que puede alcanzarse con la mano sin esfuerzo individualmente por la longitud de los brazos recibe el nombre de área de alcance. La Fig. 1.b. muestra el corte de las zonas de alcance a la altura de la superficie de la mesa. No se puede llegar con la misma facilidad a todos los lugares de esa zona de alcance. El juego de las articulaciones proporciona órbitas de movimientos más favorables y menos favorables.

Las zonas laterales son difíciles de alcanzar e innecesarias para el banco, por lo que se descartan. Quedando un área rectangular de $40 \mathrm{~cm}$ de profundidad por $120 \mathrm{~cm}$ de ancho. Al determinar la altura máxima del banco es necesario conocer la medida máxima de asimiento con el brazo extendido, la cual es de $68.5 \mathrm{~cm}$ (Fig. 2.a). La media de hombre y mujeres colombianos para el percentil 50 se presenta en las Tablas $1 \mathrm{y}$ 2.

Conociendo la altura acromial promedio para hombres y mujeres colombianos (P50), se obtiene un promedio de 132.5 $\mathrm{cm}$. Para el trabajo cómodo con el brazo extendido se toma un ángulo de $30^{\circ}$ con la horizontal, por lo que la mayor altura a la cual se trabaja con un elemento es igual a $132.5 \mathrm{~cm}+(68.5$ $\left.\mathrm{cm} \times \operatorname{sen} 30^{\circ}\right)=166.8 \mathrm{~cm} \approx 167 \mathrm{~cm}$, Fig. 2. b.

\section{F. Diseño del tablero de trabajo.}

El tablero de trabajo es diseñado con base en las medidas obtenidas a partir de las consideraciones de ergonomía. Se evalúa un perfil de aluminio cuadrado de $1 / 2$ " x 1 1 1/2" de lado, separados entre sí a una distancia de $2 \mathrm{~cm}$ para el área de sujeción de elementos, ver Fig. 3. Por medio de ajuste forzado de láminas en forma de $\mathrm{U}$ de $2 \mathrm{~cm}$ de longitud se asegura la estabilidad de cada perfil. Esta separación es elegida debido a la existencia estandarizada del perfil $2 \mathrm{~cm}$ de lado que soporta cada elemento, obteniendo un ajuste deslizante que permite montar y desmontar cada una de las piezas de la manera más práctica posible. 


\section{a. Brazo extendido}

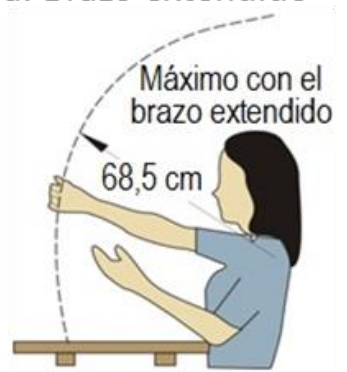

\section{b. Altura máxima}

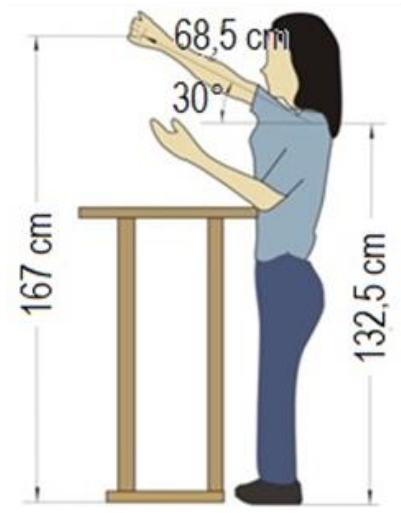

Figura 2. Alcance anterior máximo promedio (a) y altura máxima (b).

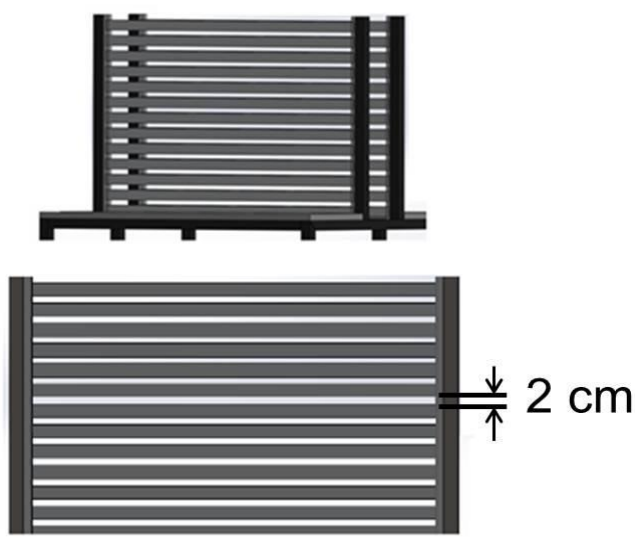

Figura 3. Perfiles del tablero de trabajo.

\section{CHEQUEO DE SEGURIDAD DE LA ESTRUCTURA}

A continuación se muestran las dimensiones del diseño base de la estructura con forma preliminar mostrada en la Fig. 4.a, al igual que todas las fuerzas y reacciones que se ven implicadas ella.

De la Fig. 4.b, la carga P resulta de la suma del peso del banco y de sus componentes, más el peso de tres estudiantes en un hipotético caso donde éstos estén sentados sobre la mesa. Siguiendo lo anterior, $120 \mathrm{~kg}$ representan la masa del banco y sus componentes, $360 \mathrm{~kg}$ sería la masa total de los estudiantes, asignando $120 \mathrm{~kg}$ para cada uno, resultando en una carga de 4709 N. RA, RB, RC y RD son las reacciones de las cuatro ruedas sobre las que va apoyado el banco. El análisis estático de fuerzas del banco, Fig. 4.b, es

$$
(\uparrow+) \sum \mathrm{F}_{\mathrm{y}}=\mathrm{R}_{\mathrm{A}}+\mathrm{R}_{\mathrm{B}}+\mathrm{R}_{\mathrm{C}}+\mathrm{R}_{\mathrm{D}}-\mathrm{P}=0,
$$

lo cual resulta en

$$
R_{A}+R_{B}+R_{C}+R_{D}=4709 N .
$$
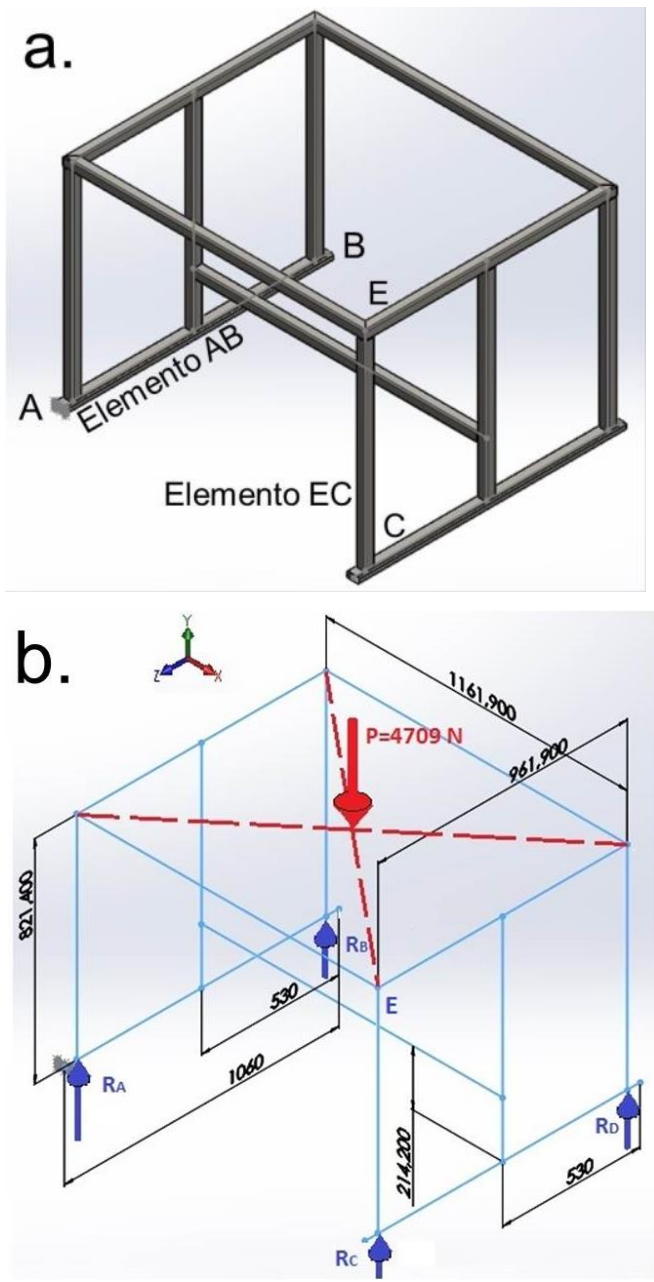

Figura 4. Distribución espacial de las fuerzas y reacciones sobre la estructura, en milímetros.

Debido a la simetría, tanto de la estructura como de las cargas, la fuerza se distribuye uniformemente a través de los seis elementos verticales, por lo cual cada uno recibe una carga dada por

$$
\mathrm{P}_{1}=\mathrm{P}_{2}=\mathrm{P}_{3}=\mathrm{P}_{4}=\mathrm{P}_{5}=\mathrm{P}_{6}=\frac{\mathrm{P}}{6}=784,8 \mathrm{~N} \approx 785 \mathrm{~N} \text {. }
$$


Se realiza el análisis de la columna EC, Fig. 5.a, con el fin de conocer los tipos de esfuerzos a los que está sometido y para encontrar la carga crítica unitaria necesaria para poner a la columna en una condición de equilibrio inestable, ya que en este estado, cualquier encorvadura pequeña del elemento o cualquier movimiento ligero del apoyo o de la carga, causaría que la columna se colapse. Se identifica el tipo de columna mediante la relación de esbeltez, la cual es regida por

$$
\mathrm{SR}=\frac{\mathrm{KL}}{\mathrm{r}}
$$

donde L: longitud real de la columna entre los puntos de apoyo o restricción lateral, K: factor de fijación de los extremos, r: radio de giro mínimo de la sección transversal de la columna, dado por

$$
r=\sqrt{\frac{I}{A}}
$$

donde A: área transversal de la misma, I: momento de inercia de la sección transversal de la columna con respecto a uno de sus ejes principales hallado mediante

$$
\mathrm{I}=\frac{\mathrm{b}^{4}}{12}-\frac{(\mathrm{b}-2 \mathrm{e})^{4}}{12},
$$

donde $\mathrm{b}=3.81 \mathrm{~cm} \mathrm{y} \mathrm{e}=0.12 \mathrm{~cm}$, son la longitud de los lados y el espesor, respectivamente, Fig. 5.b.

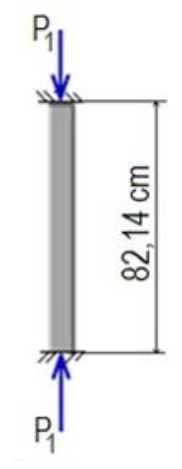

(a)DCL elemento EC

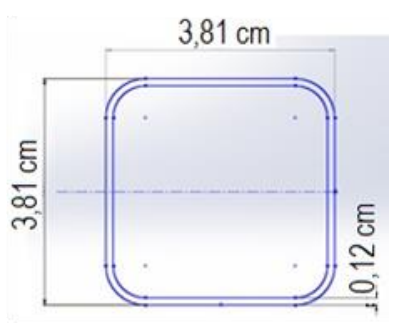

(b)Sección transversal del elemento EC
Figura 5. Diagrama de cuerpo libre y sección del elemento EC.

Se procede a hallar la relación de esbeltez, SR, tomando el factor fijación $\mathrm{K}$ correspondiente a columnas con ambos extremos fijos $\mathrm{K}=0,65$ [8], para obtener de (4) $\mathrm{SR}=32.43$. Para definir si la columna es larga, intermedia o corta se compara el valor de SR con la relación de esbeltez de transición Cc:

$$
\mathrm{C}_{\mathrm{c}}=\sqrt{\frac{2 \mathrm{E} \pi^{2}}{\mathrm{~S}_{\mathrm{y}}}}
$$

donde E: módulo de elasticidad, Sy: límite elástico. Al remplazar para acero AISI 1020 estirado en frio con módulo de elasticidad de $\mathrm{E}=200 \mathrm{GPa}$ y $\mathrm{Sy}=350 \mathrm{MPa}$ se obtiene $\mathrm{Cc}$ $=106.2$, el cual al ser comparado con el valor de (4) define la columna como intermedia. Se debe entonces usar la fórmula de J.B. Johnson [8] para la carga crítica

$$
\mathrm{P}_{\mathrm{cr}}=\mathrm{A} \sigma_{\mathrm{y}}\left[1-\frac{\sigma_{\mathrm{y}}(\mathrm{SR})^{2}}{4 \pi^{2} \mathrm{E}}\right]
$$

donde Pcr es la carga de pandeo crítica. Al sustituir en (8) se obtiene PCR $=62500000 \mathrm{~N}$, correspondiente a una carga permisible $\mathrm{P}=125000000 \mathrm{~N}$ para un factor de diseño de 5 . Esta carga permisible, en comparación con la que estaría recibiendo el elemento en cuestión, es bastante grande, por lo que se puede asegurar que no fallará. En la Fig. 6 se muestra la simulación del elemento EC realizada con Solid Works, de la cual se aprecia que el esfuerzo máximo está muy por encima del Sy del material, para un factor de seguridad de 7 , garantizando así la seguridad estructural del elemento EC.

Se procede a analizar el elemento $A B$, el cual es una de las dos barras horizontales sobre la que reposan tres fuerzas verticales hacia abajo, Fig. 4.a, cada una de aproximadamente 785 N. La Fig. 7 muestra el diagrama de cuerpo libre para este elemento, resultando en sumatoria de fuerzas dado por

$$
(\uparrow+) \sum \mathrm{F}_{\mathrm{y}}=\mathrm{R}_{\mathrm{A}}+\mathrm{R}_{\mathrm{B}}-\mathrm{P}_{1}-\mathrm{P}_{2}-\mathrm{P}_{3}=0,
$$

con sumatoria de momentos

$$
\sum \mathrm{M}_{\mathrm{A}}=-481 \mathrm{P}_{2}-962 \mathrm{P}_{3}+962 \mathrm{R}_{\mathrm{B}}=0 .
$$

Sustituyendo (9) en (10) se obtiene RB $=1177.5 \mathrm{~N}$, y al remplazar este valor en (11) se obtiene $\mathrm{RA}=1177.5 \mathrm{~N}$, ver Fig. 8.a. De la Fig. 8.b, el elemento AB está sometido a un momento flector de $188772.88 \mathrm{~N} \mathrm{~mm}$, creando esfuerzos normales en la sección transversal, mientras que la fuerza $\mathrm{V}$, Fig. 8.a, produce esfuerzos cortantes en dicha sección, domina el valor máximo del esfuerzo normal en la viga $\sigma_{\mathrm{m}}$, dado por

$$
\sigma_{\mathrm{m}}=\frac{\mathrm{M}_{\text {máx }}}{\mathrm{S}},
$$

donde $\mathrm{S}$ es el módulo de la sección. Escogiendo una viga rectangular de 2 x $11 / 16$ in de lado, material SAE 1020, Sy = $350 \mathrm{MPa}$ y $\mathrm{S}=539.2 \mathrm{~mm}^{3}$, al remplazar en (11) se obtiene un esfuerzo máximo de $\sigma_{\mathrm{m}}=63.54 \mathrm{MPa}$, para un factor de seguridad de 5.51.

La Fig. 9 presenta la simulación del elemento AB, realizada en SolidWorks, indicando un esfuerzo máximo de aproximadamente $78 \mathrm{MPa}$, para un factor de seguridad de 4.5. 


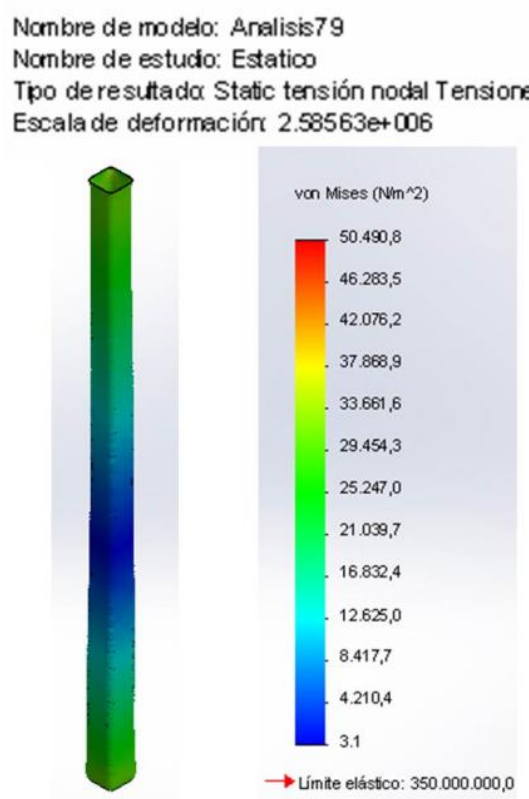

Figura 6. Simulación del elemento EC.

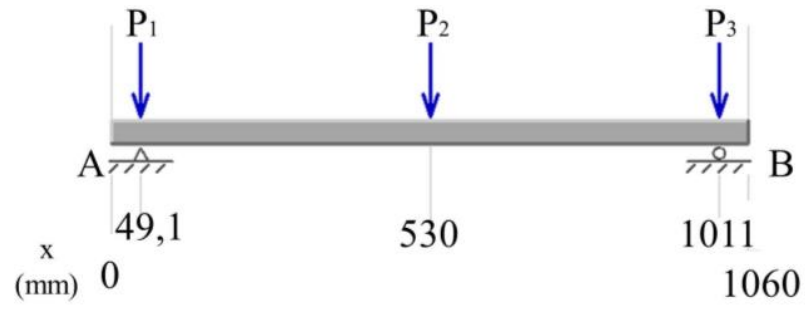

Figura 7. Diagrama de cuerpo libre del elemento AB.

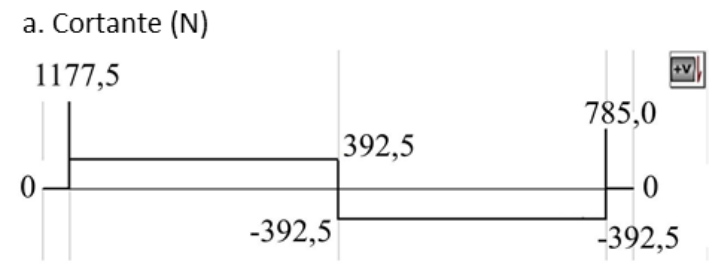

b. Momento ( $\mathrm{N} \mathrm{mm}$ )

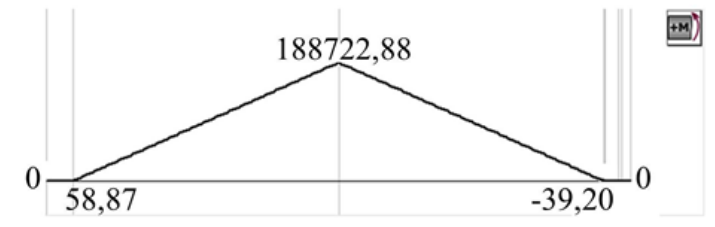

Figura 8. Diagramas del elemento AB.

La Fig. 10 presenta el banco con ruedas con freno para fácil movimiento en el laboratorio y anclaje en el sitio de trabajo. El acabado es de pintura electrostática para mayor conservación de la estructura. Se distingue un área de inserción de módulos de PLCs de diferentes marcas.

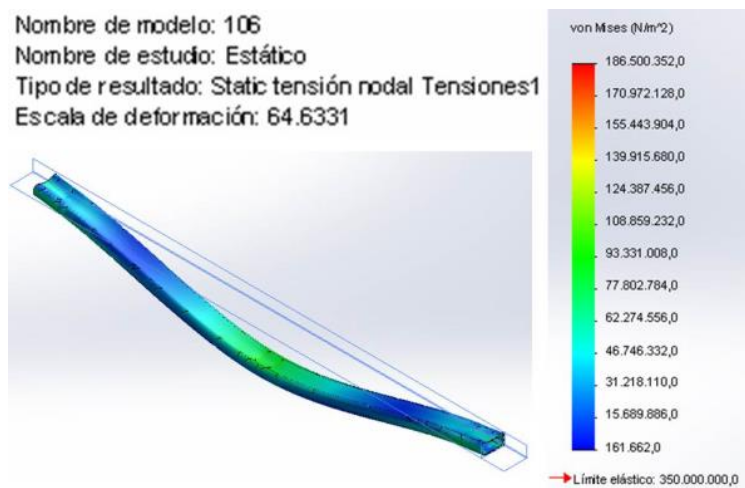

Figura 9. Simulación del elemento AB.
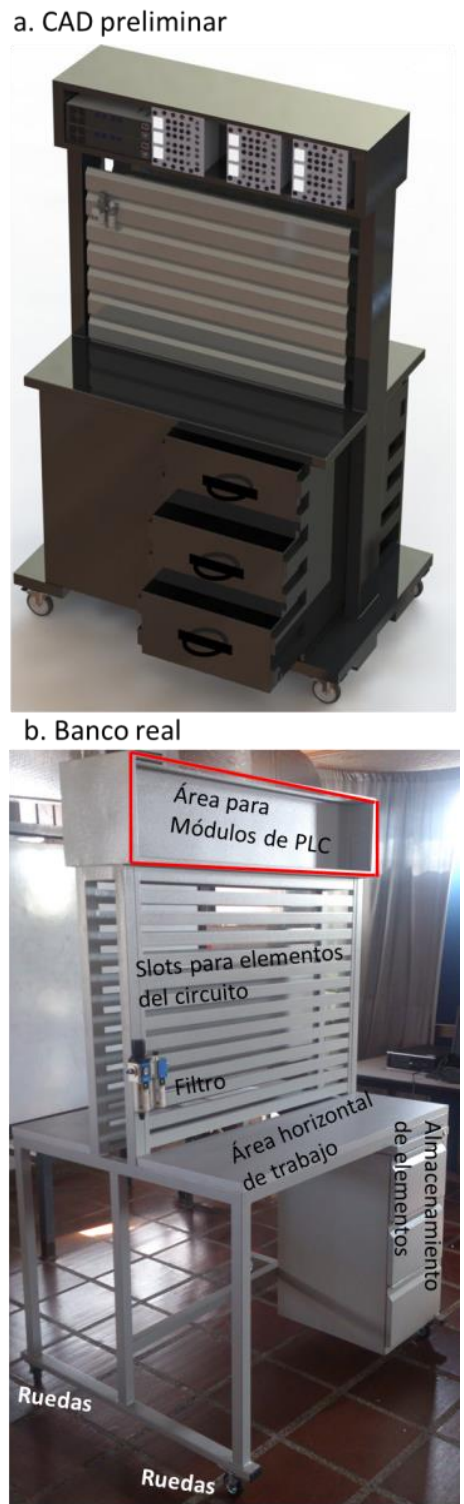

Figura 10. CAD (a) y banco construido (b). 


\section{DISPOSITIVO DE SUJECIÓN}

Para ubicar los elementos neumáticos en el tablero de trabajo del banco, se hace necesario diseñar bases con un sistema de sujeción que sea capaz de contrarrestar la fuerza que generen los componentes durante las prácticas de laboratorio, como el que se muestra en la Fig. 11.a.

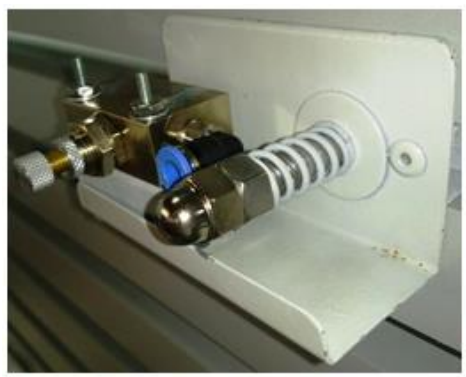

a) Sistema de sujeción montado sobre el banco

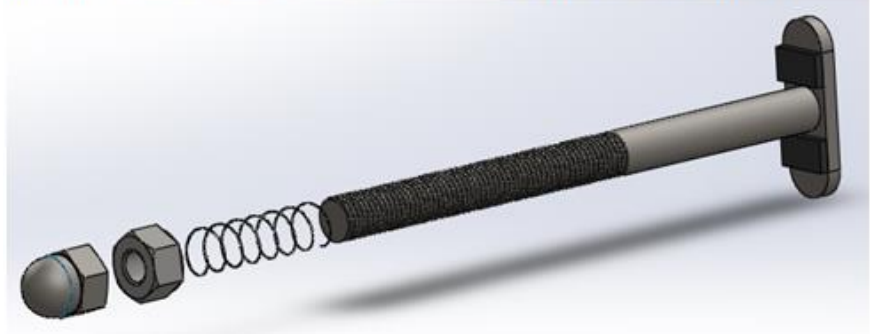

c) Vista explosionada del sistema de sujeción

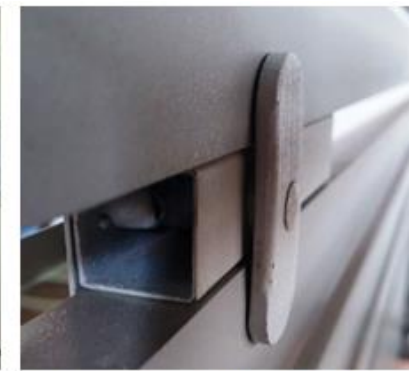

b) Parte posterior del sistema de sujeción
Figura 11. Sistema de sujeción.

Las bases están conformadas por un tubo de acero de perfil cuadrado con dimensión de dos centímetros de lado, que van ubicadas entre los perfiles del tablero de trabajo como se aprecia en la Fig. 11.b, y una placa en la que van soportadas los elementos, que varía sus dimensiones dependiendo del tamaño de éstos. El sistema de sujeción se diseña con un mecanismo de resorte, como se muestra en la Fig. 11.c, el cual posee su constante elástica $\mathrm{K}$ que se encarga de proporcionar la fuerza suficiente a la base para que no sea movida por los componentes neumáticos. El diseño del sistema de sujeción inicia determinando cuál de todos los componentes genera mayor fuerza sobre la base, siendo éste el cilindro.

La fuerza generada por el cilindro se halla con

$$
\mathrm{F}_{\text {cilindro }}=\mathrm{P} \times \mathrm{A} \text {, }
$$

donde el área es $\mathrm{A}=\pi\left(\frac{25}{2} \mathrm{~mm}\right)^{2}=490,87 \mathrm{~mm}^{2}$ y la presión del sistema $\mathrm{P}=30$ psi, para obtener Fcilindro $=101.53$ psi.

Se procede a hallar la fuerza mínima que debe generar el resorte, debido a su compresión, para producir una fuerza de fricción, entre la placa de las bases y los perfiles de aluminio del tablero de trabajo, capaz de contrarrestar la fuerza producida por los cilindros. La ecuación que rige la fuerza que

se opone al movimiento entre dos superficies en contacto está dada por

$$
\mathrm{f}_{\mathrm{s}}=\mu_{\mathrm{s}} \mathrm{N}
$$

donde fs: fuerza de fricción estática, N: fuerza normal e igual a la fuerza del resorte en este caso (Fig. 12), y $\mu$ s: coeficiente de fricción estático entre las dos superficies caucho y acero, igual a $0,9[9]$.

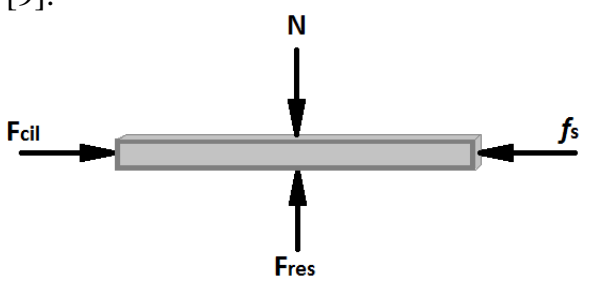

Figura 12. Diagrama de cuerpo libre (DCL) para la placa base del sistema de sujeción.

La fuerza mínima de fricción debe ser igual a la fuerza generada por el cilindro para mantenerse en equilibrio. Según el DCL de la Fig. 12 y remplazando en (13) se obtiene

$$
\mathrm{f}_{\mathrm{s}}=\mu_{\mathrm{s}} \mathrm{F}_{\text {resorte }}=\mathrm{F}_{\text {cilindro }} \text {, }
$$

de donde la fuerza del resorte requerida por el sistema de sujeción es de $112.81 \mathrm{~N}$. Se selecciona un resorte de constante $220 \mathrm{~N} / \mathrm{cm}$, y con la fuerza mínima requerida de $113 \mathrm{~N}$ se halla la distancia mínima de compresión, despejando x de (15) para $5.12 \mathrm{~mm}$. Se diseña el dispositivo para una distancia comprimida x igual a $8 \mathrm{~mm}$, excediendo la fuerza del cilindro. La Fig. 13 presenta otros elementos diversos del banco que fueron adaptados con el dispositivo de sujeción.

$$
\mathrm{K}=\frac{\mathrm{F}}{\mathrm{x}},
$$

(a) Final de carrera

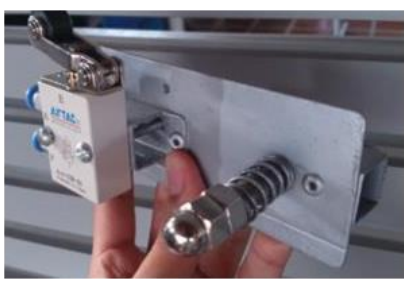

(c) Válvula $3 / 2$

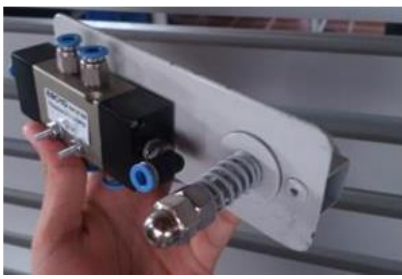

Figura 13. Algunos componentes con elementos de sujeción.

\section{(b) Válvula reguladora}

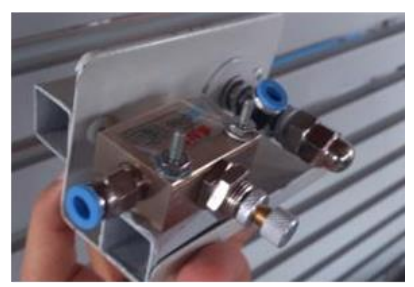

(d) Cilindro

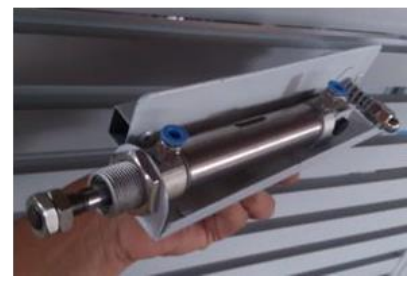




\section{PRÁCTICA DE LABORATORIO}

Se presenta una de las prácticas desarrolladas para ilustrar el uso del banco construido. Las guías se diseñaron autocontenidas para mayor comodidad y conveniencia del estudiante y profesor, con un alto nivel de detalle, para así estimular al estudiante, sobre todo en las primeras prácticas, pues es cuando más se requiere una detallada descripción de cada paso necesario para el desarrollo de la experiencia.

A. Temas de la Práctica de Laboratorio.

Mando electroneumático con válvula de una bobina y válvula doble bobina.

\section{B. Objetivos Didácticos.}

- Diseñar un circuito neumático para el control de dos cilindros de doble efecto controlados por electroválvulas.

- Usar sensores y/o finales de carrera como señales de entrada al PLC para establecer una programación.

C. Descripción del Problema.

Una estación de transferencia consta de dos cilindros, 1A y 2A, Fig. 14, que se encargan de trasladar discos desde un almacén hacia una estación de proceso. El cilindro 1A empuja los discos fuera del almacenamiento hacia el cilindro $2 \mathrm{~A}$, el cual lo desvía hacia su destino final como se muestra en la Fig. 14. Se requiere diseñar un circuito que permita controlar el movimiento automático de los cilindros sabiendo que el vástago del cilindro $2 \mathrm{~A}$ sale una vez que el vástago del cilindro 1A llega a su posición final. Además, el sistema debe controlarse como ciclo único y como ciclo continuo.

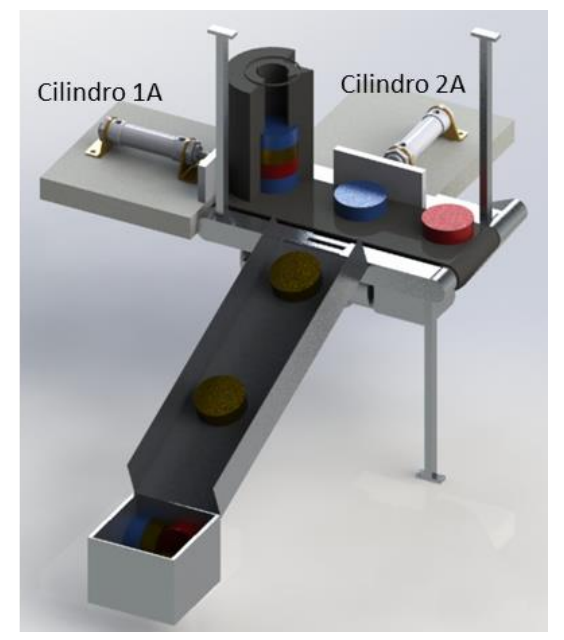

Figura 14. Sistema de la banda transportadora.

A continuación el lector será guiado a través de una serie de pasos detallados que le permitirán desarrollar las soluciones propuestas en esta práctica de laboratorio.
D. Diseño del Automatismo Cableado.

Se presentan los circuitos correspondientes a la solución del problema enunciado. La Fig. 15 muestra el diagrama de movimientos del sistema, mientras que la Fig. 16 presenta una posible solución para el problema. Para el desarrollo de la práctica se necesitan los elementos listados en la Tabla 3. El correspondiente circuito eléctrico se presenta en la Fig. 17.

\section{E. Montaje del Circuito en el Banco de Trabajo.}

Siguiendo el esquema del circuito neumático presentado en la Fig. 16, los componentes se dispondrán en el tablero del banco de trabajo utilizando el sistema de sujeción. A continuación se realizan las correspondientes conexiones entre elementos para crear el circuito mostrado en la Fig. 18; mientras que la Fig. 19 muestra las conexiones en el PLC. Las guías cuentan con un video de ilustración para la creación paso a paso del circuito.

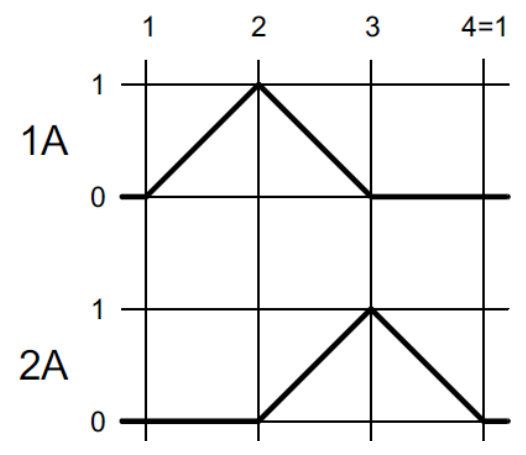

Figura 15. Diagrama de movimientos.

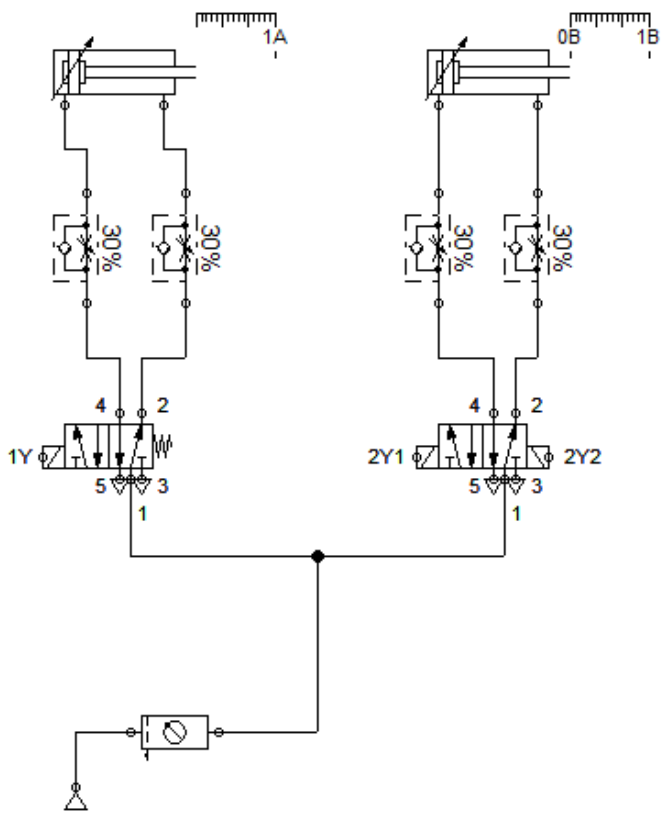

Figura 16. Diagrama de circuito neumático. 
El PLC se comunica con el computador por medio del puerto USB. El cable de comunicación se conecta en la entrada MPI del PLC y luego a la entrada USB del ordenador.

\begin{tabular}{|l|l|l|}
\hline Item & Cant. & Descripción \\
\hline 1 & 2 & Cilindro de doble efecto \\
\hline 2 & 1 & Electroválvula $5 / 2$ monoestable \\
\hline 3 & 1 & Electroválvula 5/2 biestable \\
\hline 4 & 4 & Válvula reguladora de flujo \\
\hline 5 & 1 & Sensor inductivo PNP \\
\hline 6 & 2 & Final de carrera \\
\hline 7 & 1 & Juego de mangueras \\
\hline
\end{tabular}

Tabla 3. Lista de componentes para la práctica.

\section{F. Diseño del Automatismo Programado.}

El paso siguiente es diseñar el programa para luego cargarlo al PLC. Este diseño se realiza por medio del software Administrador SIMATIC. Información detallada acerca de la programación y demás aspectos puede ser encontrada en la referencia [10].

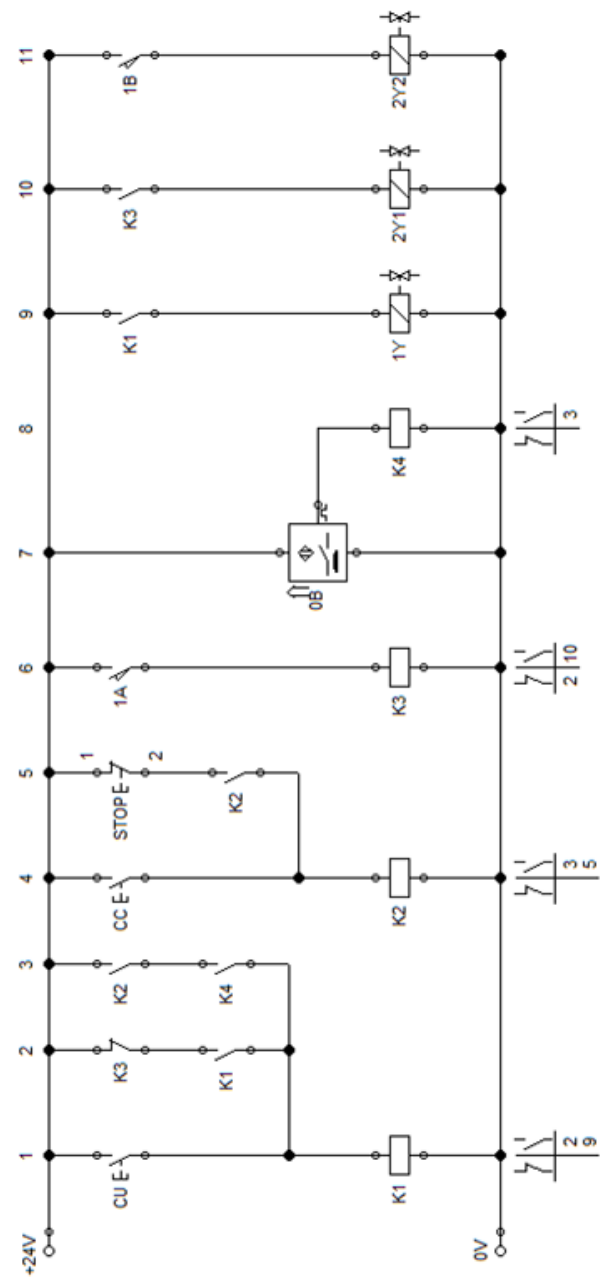

Figura 17. Circuito eléctrico del sistema.

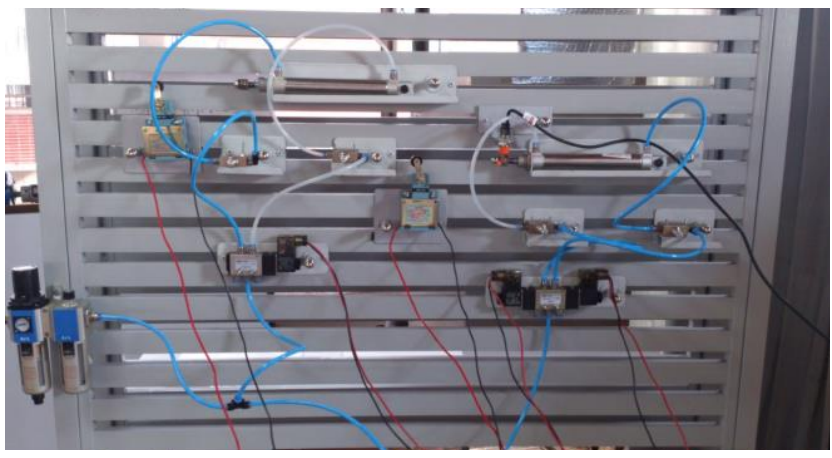

Figura 18. Montaje del circuito neumático en el tablero de trabajo.

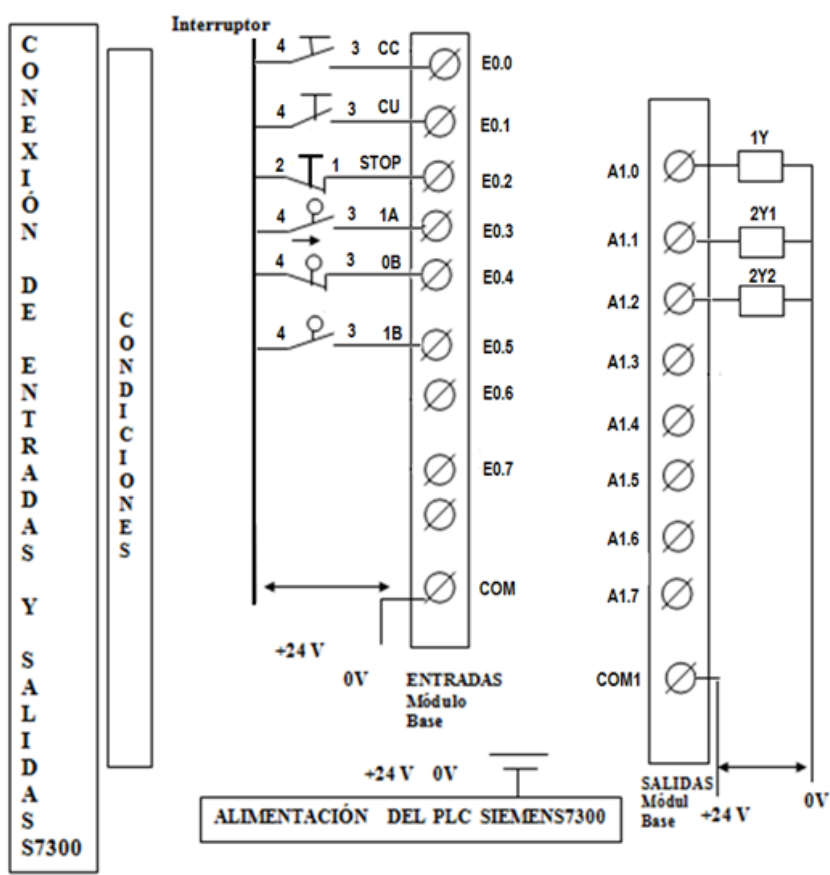

Figura 19. Conexión de entradas y salidas del PLC.

\section{CONCLUSIÓN}

Se diseñó y construyó un banco de pruebas para prácticas en las áreas de neumática, automatización y control, diseñado como una estructura que soporta todos los accesorios en el panel y simulado con un peso hipotético (abuso) de tres estudiantes entados en el área de trabajo. Las dimensiones de dicha estructura fueron determinadas fueron determinadas de manera que existiese armonía dimensional entre el personal que utilizará el banco, el banco mismo y los objetos que se integrarían al último, para lo cual se utilizaron criterios de ergonomía en el diseño del producto, para además evitar la fatiga muscular en el uso del banco en las prácticas de laboratorio. 
Los elementos electroneumáticos se fijaron a un sistema de sujeción dimensionado de acuerdo con las cargas de trabajo del circuito, permitiendo además de un fácil desarrollo de las prácticas, flexibilidad en los circuitos a diseñar gracias a su rápido y seguro montaje y desmontaje. Las bases de sujeción de los elementos neumáticos fueron construidas de manera que ocupen el menor espacio en el tablero, sean manejables y a la vez proporcionen una sujeción firme y segura.

Como complemento al correcto uso del banco, se elaboraron guías prácticas autocontenidas con el fin de facilitar el aprendizaje y la familiarización del estudiante a los diferentes elementos que conforman un circuito neumático, así como también su programación, ensamble, puesta en marcha y operación. Se diseñaron para que el estudiante evidencie los conceptos teóricos presentados en clase por el docente e instarlo al análisis de situaciones problema que se pueden presentar en estos circuitos. Ayudas audiovisuales fueron creadas para que el mínimo detalle sea apreciado y entendido claramente.

\section{AGRADECIMIENTOS}

Los autores agradecen el apoyo recibido por parte de la Vicerrectoría de Investigaciones, Extensión y Proyección Social de la Universidad de Atlántico para el desarrollo de este proyecto.

\section{REFERENCIAS}

[1]. Asociación Colombiana de Ingenieros Eléctricos, Mecánicos y Afines, ACIEM. Áreas de Formación de los Ingenieros, Capítulo 10: Formación específica de los ingenieros mecánicos, 2006.

[2]. A. Creus. Neumática e Hidráulica. Alfa omega Grupo Editor, S.A. de C.V., México, 2007.

[3]. A. Guillén. Introducción a la Neumática. MARCOMBO, S.A., 1988.

[4]. L. Tortosa, C. Garcia and A. Ferreras, Ergonomia y Discapacidad. Valencia: Instituto de Biomecánica de Valencia (IBV). 1999.

[5]. Definition and Domains of ergonomics. IEA International Ergonomics Association. [En línea] 2013. http://www.iea.cc/whats/index.html.

[6]. P. Mondelo, E. Gregori y P. Barrau, Ergonomía 1 Fundamentos. 3 ed. Barcelona. Edicions UPC, 1994. $192 \mathrm{p}$.

[7]. J. Estrada, J. Camacho, M. Restrepo y C. Parra, "Parámetros Antropométricos de la Población Laboral Colombiana 1995". En: Revista Facultad Nacional de Salud Pública, 1998; p. 112-139.

[8]. R. Budynas y J. Nisbett. Diseño en Ingeniería Mecánica de Shigley. 8 ed. Interamericana: McGraw-Hill, 2008. 1096 p.

[9]. J. Wilson y A. Buffa, Física. 5 ed. México: Pearson Educación, 2003. 842 p.

[10]. Autores removidos para evaluación a ciegas, "Diseño y Construcción de un Banco de Pruebas de Entrenamiento para Prácticas Electroneumáticas," Tesis Ing. Mecánico, Universidad del Nombre removido para evaluación a ciegas, Colombia, 2014. 